\title{
Noncollinear parametric amplification in the near-infrared based on type-II phase matching
}

\author{
C Schmidt, J Bühler, A-C Heinrich, A Leitenstorfer and D Brida \\ Department of Physics and Center for Applied Photonics, University of Konstanz, D 78457 Konstanz, \\ Germany \\ E mail: daniele.brida@uni konstanz.de
}

\begin{abstract}
Noncollinear parametric amplification based on type-II phase matching for the generation of ultrabroadband and tunable spectra in the near infrared is investigated. In a noncollinear geometry the group velocity matching condition between signal and idler can be obtained in frequently used crystals such as $\beta$-barium borate (BBO) even for wavelengths fully located in the anomalous dispersion region. The extremely broadband operation, peculiar tuning possibilities and straightforward experimental implementation with the standard BBO crystal pave the way for a versatile NIR source in ultrafast spectroscopy.
\end{abstract}

Keywords: ultrafast optics, parametric processes, nonlinear optics

\section{Introduction}

The generation of ultrabroadband, few-cycle pulses is at the center of modern optics [1]. Pulses characterized by durations limited to few femtoseconds allow the investigation and the control of fundamental phenomena occurring in matter at their characteristic timescale such as phase transitions [2, 3], energy transfer [4], quasi-particle formation [5, 6], charge carrier relaxations [7] and many other chemical or biological problems [8, 9]. Additionally, the extremely short duration of intense electromagnetic waves allows non-perturbative excitation of matter [10] with, for example, the emission of high order harmonics [11]. Here we present a simple and flexible concept for the generation of few-cycle pulses in near-infrared. This novel source is based on noncollinear parametric amplification and can be employed in ultrafast spectroscopy with perspectives for its scaling to high-energy applications.

A number of techniques for the synthesis of ultrashort pulses with different properties and specifications have been developed in the last years. The most straightforward approach for the generation of few-cycle pulses relies on the broad gain bandwidth of Ti:sapphire oscillators and subsequent amplifiers operating at the wavelength of $800 \mathrm{~nm}$ [12]. However, to extend the tunability of ultrafast sources, it is common to rely on nonlinear optics for broadband frequency conversion. Methods based on third-order nonlinearities, that allow mixing processes in bulk materials [13] or in highly-nonlinear [14] and photonic crystal fibers, offer extremely broadband and highly stable output pulses at moderate energy levels. This process can be scaled to high power with the use of a gas medium contained in a hollow fiber [15] or exploited for filamentation [16]. Nevertheless, the ultimate flexibility for an ultrafast source can be obtained by optical parametric amplification (OPA) [17]. This second-order nonlinear process allows the generation of tunable ultrashort pulses spanning from UV to far-IR and $\mathrm{THz}$ radiation [18 20]. OPAs are workhorses in ultrafast spectroscopy [21] when employed at low power and can easily be scaled up to high powers for extreme field applications [22 26]. In this work, we discuss how OPAs can be designed to operate in the near-IR spectral region for the production of broadband and tunable pulses with a geometry that employs noncollinearity [27] combined with type-II phase matching. Type-I noncollinear OPAs (NOPA) in the near-IR are limited to nonlinear materials with high refractive index 


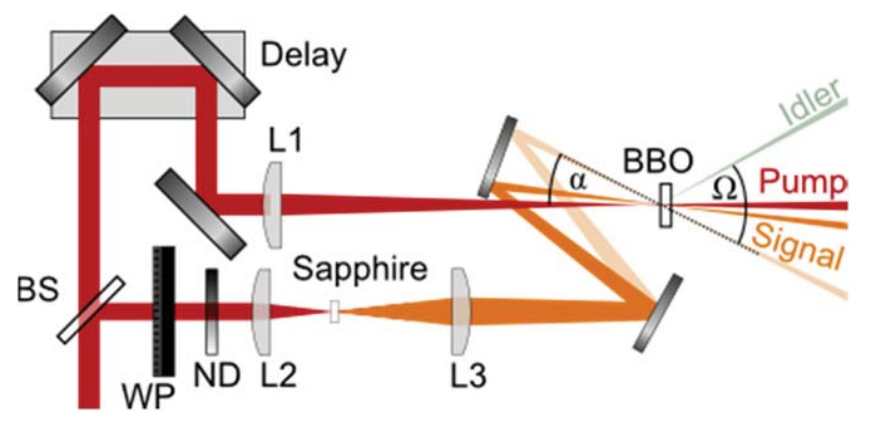

Figure 1. Schematic setup of a noncollinear optical parametric amplifier with $\alpha$ being the noncollinearity angle and $\Omega$ the angle between signal and idler. $\alpha$ can be aligned to optimize the phase matching condition. BS: beam splitter, $R=10 \%$, WP: $\lambda / 2$ waveplate, $\mathrm{ND}$ : linear neutral density filter, L1: pump focusing lens, $300 \mathrm{~mm}$; L2: lens with $75 \mathrm{~mm}$ focal length, L3: lens with $50 \mathrm{~mm}$ focal length; BBO: $\beta$ barium borate or other suitable nonlinear crystals.

[28 32], i.e. with zero dispersion wavelength exceeding $1600 \mathrm{~nm}$ such as potassium niobate or lithium tantalate. Instead, the conditions discussed in this work can be achieved in virtually any nonlinear crystal including $\beta$-barium borate (BBO). This fact provides additional flexibility in terms of tunability and efficiency to the OPA process.

\section{Optical parametric amplification}

Parametric amplification is generally employed as a front-end of a Ti:sapphire femtosecond amplified laser system operating at $\mathrm{kHz}$ repetition rate. Recently, also $\mathrm{Yb}$ lasers are gaining appeal as driving sources [33]. The conceptual scheme for the realization of an OPA is shown in figure 1. A white-light supercontinuum is generated via self-phase modulation by focusing a small portion of the driving pulse into a few-mmthick plate made of a $\chi^{3}$ nonlinear medium. This seed acts as the signal in the amplification process that takes place in a $\chi^{2}$ nonlinear crystal, in our case BBO. The OPA is pumped by the fundamental or second harmonic of the laser system output at 800 or $400 \mathrm{~nm}$, respectively. During amplification an idler beam is generated due to energy conservation. The momentum conservation condition, i.e. phase matching, determines which spectral components of the supercontinuum can be effectively amplified.

The key ingredient for the generation of a few-cycle pulse with an OPA is optimum group velocity matching between signal and idler [17]. If these two pulses propagate with the same speed, the energy is efficiently exchanged over a broad bandwidth during the nonlinear amplification process. In detail, we evaluate the group velocity mismatch $(\mathrm{GVM})$ by defining a parameter $\delta$ that is proportional to the difference of the reciprocal of the signal $\left(v_{\mathrm{gs}}\right)$ and idler $\left(v_{\mathrm{gi}}\right)$ group velocities:

$$
\delta=\frac{1}{v_{\mathrm{gs}}}-\frac{1}{v_{\mathrm{gi}}} .
$$

To the first order, the gain bandwidth is proportional to the inverse of the $\delta$ parameter as can be calculated by solving the nonlinear coupled equations that describe the parametric amplification process in a $\chi^{2}$ crystal [17]. When the group velocities are matched, i.e. $\delta=0$, the bandwidth is only limited by higher orders such as group delay dispersion that start to be significant.

The group velocity matching condition between signal and idler can be easily achieved in type-I configurations owing to the fact that signal and idler share the same dispersion relation for the refractive index. In particular, it is possible to: (i) have degenerate amplification so that signal and idler are both at approximately half of the pump photon energy and thus propagating with the same dispersion of refractive index [34 36]; (ii) have signal and idler at frequencies symmetric with respect to the zero group velocity dispersion point, i.e. one propagating in the normal and the other in the anomalous dispersion region so to match their velocities [37]. A third important approach relies on the socalled noncollinear geometry [38, 39]. In this case, the requirement of group velocity matching can be obtained when the idler is faster than the signal. Under this condition, it is possible to introduce an angle $\alpha$ between signal and pump so that the corresponding idler is generated at the new angle $\Omega$, thus preserving the total momentum:

$$
k_{\mathrm{p}} \cos \alpha=k_{\mathrm{s}}+k_{\mathrm{i}} \cos \Omega .
$$

In this geometrical setup the component of the idler velocity is projected along the signal direction

$$
k_{\mathrm{p}} \sin \alpha=k_{\mathrm{i}} \sin \Omega
$$

and allows for GV matching:

$$
v_{\mathrm{gs}}=v_{\mathrm{gi}} \cos \Omega
$$

The concept of NOPAs based on a type-I phase matching condition has been employed for a variety of different crystals and spectral regions [40 42]. As an example, the prototypical crystal for parametric amplification, BBO, cut at phase matching angle $\theta=32^{\circ}$, provides a remarkable phase matching bandwidth extending from 500 to $700 \mathrm{~nm}$ when pumped by the second harmonic of a Ti:sapphire laser and with a noncollinearity angle of $3.7^{\circ}[38,39]$. Recently it has been demonstrated that the NOPA concept can be extended to nearIR wavelengths by exploiting nonlinear crystals with high refractive index [21, 43, 44]. These materials, in fact, provide a red-shifted dispersion curve and zero-dispersion point so that the idler can display a larger group velocity than the signal, a condition that cannot be achieved in BBO with $800 \mathrm{~nm}$ pumping. In particular, the noncollinearity concept can be exploited in quasi phase matching condition with periodically poled crystals. In this case the amplification bandwidth relies on group velocity matching analogously to the standard type-I geometry. It has also been shown that KTP, a high refractive index crystal, can support noncollinear operations while employing type-II phase matching [45] in an OPA pumped at $800 \mathrm{~nm}$. 


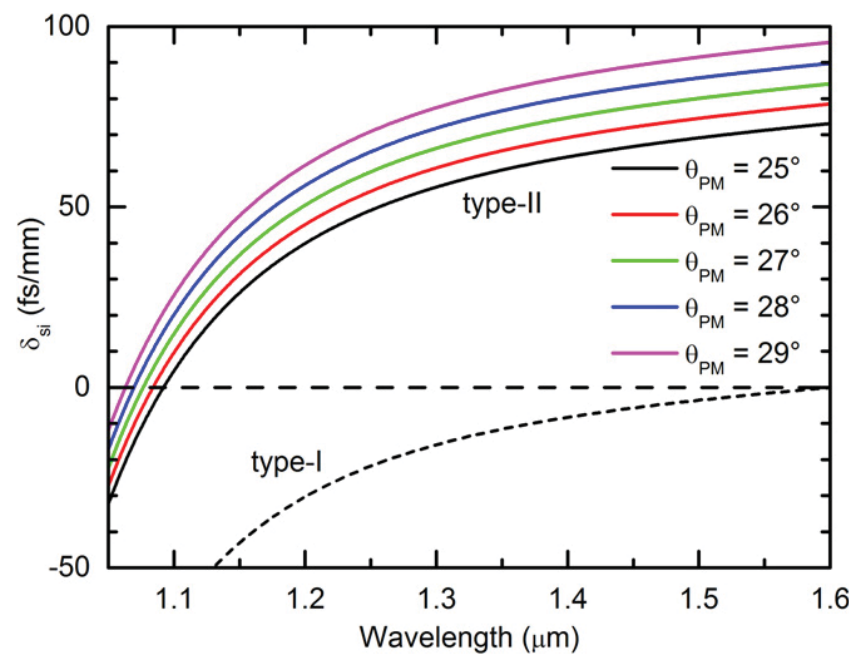

Figure 2. Calculated group velocity mismatch $\delta$ between signal and idler pulse for a $800 \mathrm{~nm}$ pumped NOPA based on BBO. The curves depict the values for type I interaction and for the interesting cases of type II interactions for different phase matching angles in the case of collinear geometry. Angles $\theta$ from $25^{\circ}$ to $29^{\circ}$ correspond to signal amplification optimized to be centered from 1150 to $1600 \mathrm{~nm}$, respectively.

\section{Noncollinear parametric amplifier with type-II phase matching}

Interestingly, BBO fully allows the beneficial scheme of noncollinear amplification when signal and idler propagate along different optical axes of the birefringent medium in type-II conditions. This feature is allowed by the stronger dispersion occurring for propagation along the ordinary axis, with $\mathrm{BBO}$ being a negative uniaxial crystal. Using a broadband near-IR seed propagating on the ordinary plane (o) with pumping at $800 \mathrm{~nm}$ (e), the idler is generated on the extraordinary axis $(\mathrm{o}($ signal $)+\mathrm{e}($ idler $)=\mathrm{e}($ pump $))$.

Figure 2 shows the GVM for type-II phase matching compared to type-I in the case of $800 \mathrm{~nm}$ pumping. The $\delta$ parameter is positive only in the type-II case. Therefore, it satisfies the precondition for a noncollinear amplification scheme.

The first difference between a type-I and a type-II NOPA consists in the fact that $\delta$ is not unambiguous anymore and depends on the phase-matching angle $\theta$ of the BBO crystal. In fact, a change in $\theta$ is also reflected in the refractive index for the idler beam affecting its propagation. The phase matching condition relies on implicit functions for the calculation of the angles $(\theta, \alpha$, and $\Omega)$ that can be easily solved numerically:

$$
\begin{gathered}
k_{\mathrm{p}}(\theta) \cos \alpha=k_{\mathrm{s}}+k_{\mathrm{i}}(\theta) \cos \Omega, \\
k_{\mathrm{p}}(\theta) \sin \alpha=k_{\mathrm{i}}(\theta) \sin \Omega .
\end{gathered}
$$

The second conceptual difference relies on the existence of two distinct type-II NOPA geometries. The noncollinearity angle becomes dependent on the direction of the beams with respect to the optical axis of the BBO crystal (see figure 3) with impact on the idler propagation. The pump propagates in the same direction of the crystal in both cases. The signal is (a)

$\alpha>0$

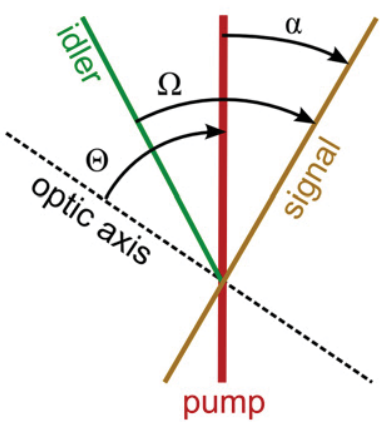

(b)
Figure 3. Two possible implementations of the noncollinear phase matching with respect to the crystallographic axis of a uniaxial material: (a) the signal is injected at a larger angle with respect to the optical axis, (b) opposite situation. The two conditions are not symmetric and, as discussed in the text, do not lead to the same amplification conditions. In this sketch the signal is polarized out of plane while pump and idler are in plane.

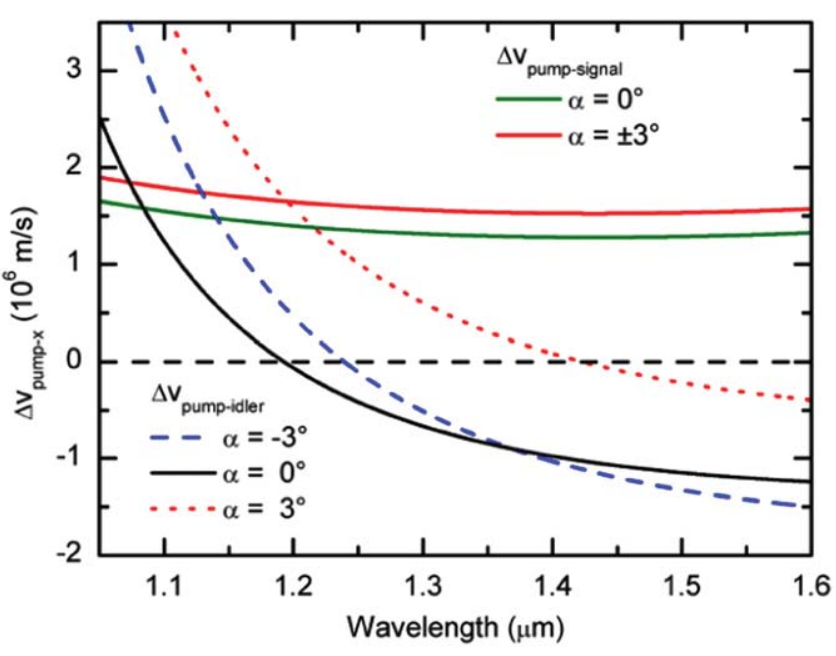

Figure 4. Calculated group velocity difference in BBO crystal of signal or idler with respect to the pump beam centered at $800 \mathrm{~nm}$. The graph shows the calculation for various noncollinearity angles at $\theta=26^{\circ}$ : the pump is faster than the signal but slower than the idler for negative values of $\alpha$.

ordinary, so it is not affected in its propagation but can be injected in two alternative directions while keeping a noncollinear interaction with the pump. Thus, the extraordinary polarized idler can be generated at two specific angles with respect to the optical axis characterized by propagation with different group velocity. The result is the existence of two phase matching conditions. In our notation case (a), as reported in figure 3, is defined for positive values of $\alpha$ and (b) for negative values.

Another important aspect is the higher efficiency that can be obtained with type-II interaction. Even if the nonlinear coefficient is lower due to crystallographic reasons, standard type-II amplification allows the exploitation of group velocity of signal and idler to achieve maximum conversion efficiency 


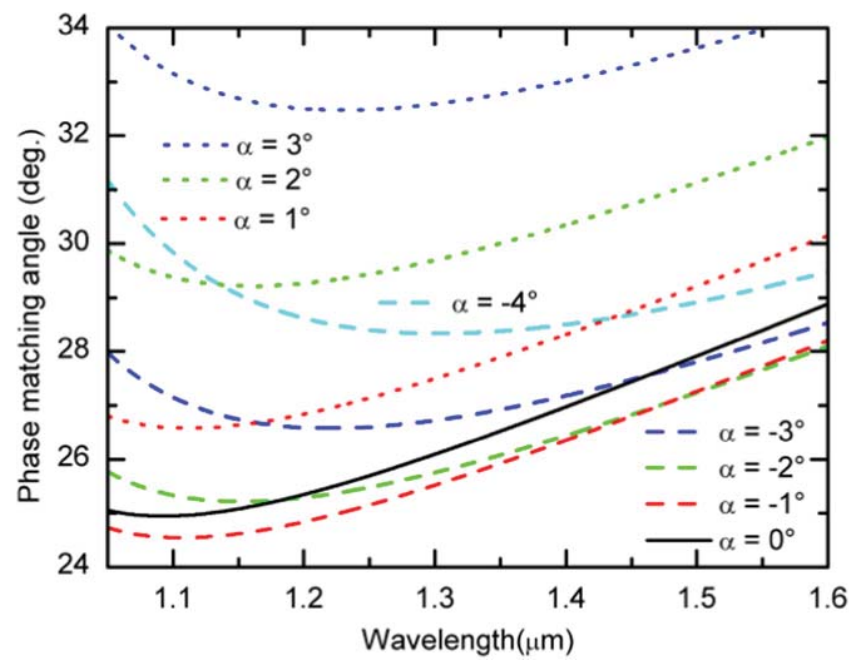

Figure 5. Calculated phase matching angle $\theta$ for type II interaction in $\mathrm{BBO}$ as a function of signal wavelength and pump signal angle $\alpha$. Pump wavelength is fixed at $800 \mathrm{~nm}$.

from the pump. With respect to the pump pulse the signal is slower while the idler is faster as plotted in figure 4 . This interplay mechanism traps the three beams via the nonlinear process thus maximizing the conversion efficiency [46]. The favorable condition is still possible with a noncollinear phase matching geometry with type-II phase matching and it is significant in particular for the configuration with negative values of $\alpha$. It should be noticed that this configuration results in a residual spatial walk-off of $3.5^{\circ}$ and $3.63^{\circ}$ for pump and idler while full compensation can be achieved with positive values of $\alpha$.

The implicit dependence of the parameters describing type-II noncollinear amplification gives some peculiar features to the OPAs in terms of bandwidth and tunability. This fact is demonstrated in figure 5 that displays the calculated phase matching angle of BBO as a function of signal wavelength and internal pump-signal angle $\alpha$. The phase matching condition considered is pump $(800 \mathrm{~nm}-\mathrm{e})=$ signal (o) + idler (e).

In case of collinear amplification $\left(\alpha=0^{\circ}\right)$, as expected, the phase matching angle varies rather steeply with respect to the signal wavelength thus limiting the maximum amplification bandwidth. By introducing the noncollinearity between pump and signal, the dependence becomes flatter thus relaxing the phase matching condition on the BBO cut angle $\theta$. When $\alpha$ is set between $-4^{\circ}$ and $+3^{\circ}$, we can obtain a broad gain bandwidth for the seed amplification with $\theta$ becoming less dependent on the signal wavelength. Interestingly, it is possible to combine this condition with tunability. In fact, the flat point of the phase matching curve shifts towards longer wavelengths by increasing the absolute value of $\alpha$. This feature is not achievable with type-I NOPA where the gain bandwidth is strictly centered at a specific spectral region for a given pump frequency. In this case tunability is obtained only at the expense of strongly shaping the output spectrum.

Again, the type-II NOPA geometry with negative $\alpha$ shows some intrinsic benefits: (i) the $\mathrm{BBO}$ crystal cut $\theta$ does

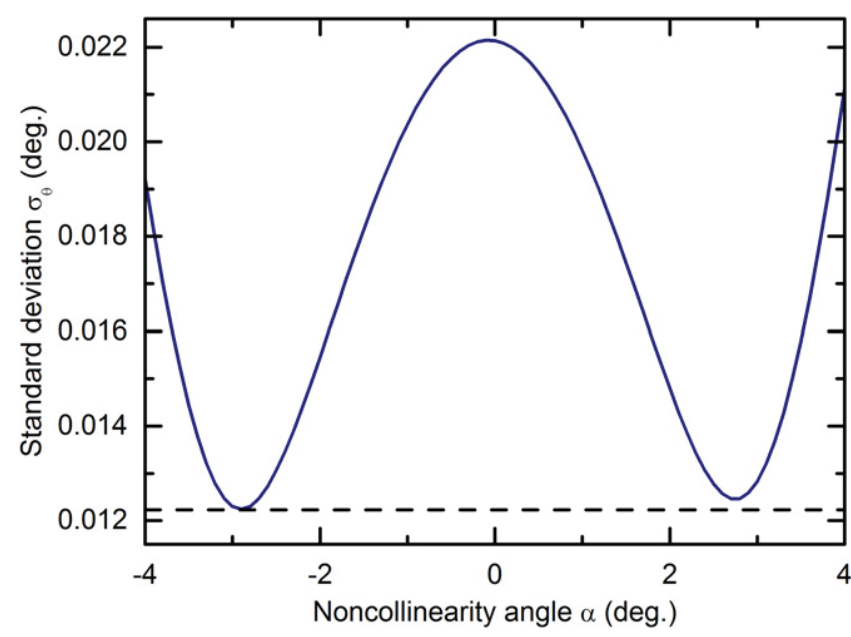

Figure 6. Standard deviation of the phase matching curve, between $1.05 \mu \mathrm{m}$ and $1.6 \mu \mathrm{m}$, in dependence of noncollinearity angle $\alpha$. The curve shows a slight asymmetry in the phase matching condition as determined by the positive or negative values of the angle $\alpha$.

not depend significantly on the specific choice of $\alpha$, allowing the use of a single BBO crystal and minor tweaks in the OPA alignment for the optimization of the signal frequency and the corresponding bandwidth; (ii) the configuration leads to slightly better results in terms of maximum bandwidth that can be achieved. Figure 6 reports the integrated deviation of the phase matching curve with respect to the target frequencyindependent condition, i.e. flat phase matching curve. This figure of merit is a good indication on how broad the phase matching condition is. Its shape is slightly asymmetric. The broadest pulses can be achieved for negative values of $\alpha$.

It should be noted that the maximum bandwidth calculated for type-II noncollinear phase matching is not as broad as achievable with a type-I NOPA based on a high refractive index nonlinear crystal. Nevertheless, this geometry introduces two important aspects along with the possibility to generate few-cycle pulses in the near-IR: (i) increased tunability with preservation of the spectral shape, which is important in applications where spectral selectivity must be combined with extreme temporal resolution, e.g. in ultrafast spectroscopy, and (ii) easier power scalability owing to the higher conversion efficiency and BBO robustness at high pumping levels.

\section{Experimental realization}

To demonstrate the high gain bandwidth and the flexibility of the type-II noncollinear phase matching design we implemented a NOPA pumped by the fundamental radiation provided by a Ti:sapphire amplified laser (150 fs at $780 \mathrm{~nm}$ central wavelength). The setup follows the general sketch reported in figure 1. It is seeded by white-light supercontinuum generated in a $4 \mathrm{~mm}$ thick sapphire plate. Amplification takes place in a $2.5 \mathrm{~mm}$ thick BBO crystal in type-II configuration (cut at $\theta=29^{\circ}$ ). The $150 \mu \mathrm{J}$ pump pulse is focused with a $300 \mathrm{~mm}$ focal length lens. The crystal is placed 


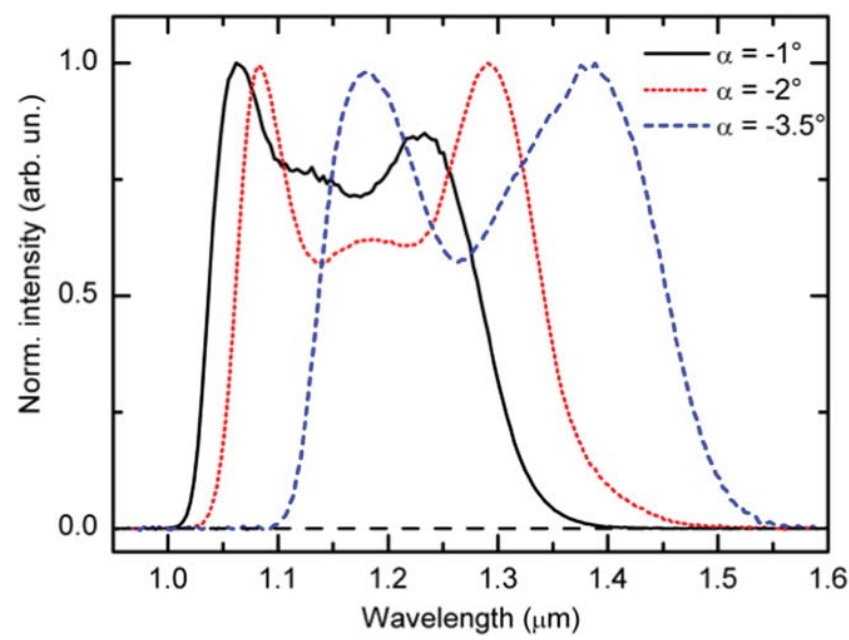

Figure 7. Spectra of the pulses generated by a noncollinear type II phase matched OPA for different internal pump signal angles $\alpha$. For the black spectrum $\alpha$ is chosen to be $-1^{\circ}$ internal, for the red one $\alpha=-2^{\circ}$ and for the blue one $\alpha=-3.5^{\circ}$. The respective transform limited pulse duration are $12.5 \mathrm{fs}(20 \mu \mathrm{J}$ pulse energy $), 11.1 \mathrm{fs}$ $(15 \mu \mathrm{J})$ and $12 \mathrm{fs}(10 \mu \mathrm{J})$, respectively.

$50 \mathrm{~mm}$ behind the focal plane, where the peak intensity is approximately $200 \mathrm{GW} \mathrm{cm}^{-2}$. The direction of the signal beam can be easily varied by means of the two steering mirrors preceding the nonlinear crystal. Although modematching of seed and pump in the crystal is not extremely critical, owing to the loose focusing condition, the spatial overlap can be optimized by adjusting the refocusing lens (L3 in figure 1) after changing the signal-pump angle. The pulse energies achieved in a single stage amplifier exceeds $10 \mu \mathrm{J}$ with $150 \mu \mathrm{J}$ pump energy.

The spectra shown in figure 7 prove the broadband operations of the type-II near-IR NOPA based on BBO. The resulting FWHM are 250, 280 and $320 \mathrm{~nm}$ for increasing angles respectively, corresponding to a frequency bandwidth of $60 \mathrm{THz}$. The signal spectra are close to the zero dispersion point of BBO and fused silica, thus the phase accumulated in the OPA stage is negligible. The residual spectral phase is mostly inherited from the white-light generation process itself. This chirp can be temporally compressed by means of a pair of glass prisms, resulting in close to transform-limited pulse duration. The experimental measurements demonstrate that the broadband pulses can be tuned without significant shaping of their spectra even at saturation.

\section{Conclusions}

In conclusion, we have introduced the concept of using the prototypical nonlinear crystal BBO for broadband noncollinear amplification in near-IR thanks to type-II phase matching. This NOPA offers additional features with respect to the standard type-I designs based on high refractive index materials. On one hand it provides tunability capabilities while preserving ultrabroadband operations. On the other hand it is based on a robust material that allows scalability to high power with multiple amplification stages with suitable pulse-tilting techniques to compensate for the noncollinear geometry at large spot sizes [47]. This source is also an ideal candidate to be employed in coherent synthesis schemes for the generation of single-cycle pulses [14, 48].

At low power levels, the near-IR NOPA is readily available for ultrafast spectroscopy applications where it can combine ultrashort pulse generation for few-fs temporal resolution with the tunability required to precisely address specific optical transitions.

\section{Acknowledgments}

D B acknowledges the support of the Emmy Noether Program of the Deutsche Forschungsgemeinschaft (DFG), of Zukunftskolleg and of EC through the Marie Curie CIG project 'UltraQuEsT' $\mathrm{n}^{\circ}$ 334463. A L acknowledges the European Research Council Advanced Grant 'UltraPhase' (ERC-2011-AdG No. 290876).

\section{References}

[1] Kärtner F X 2004 Few Cycle Laser Pulse Generation and its Applications (Topics in Applied Physics vol 95) (Berlin: Springer)

[2] Dal Conte S et al 2015 Snapshots of the retarded interaction of charge carriers with ultrafast fluctuations in cuprates Nat. Phys. 114216

[3] Mayer B et al 2015 Tunneling breakdown of strongly correlated insulating state in $\mathrm{VO}_{2}$ induced by intense multi terahertz excitation Phys. Rev. B 91235113

[4] Falke S M et al 2014 Coherent ultrafast charge transfer in an organic photovoltaic blend Science 34410015

[5] Huber R, Tauser F, Brodschelm A, Bichler M, Abstreiter G and Leitenstorfer A 2001 How many particle interactions develop after ultrafast excitation of an electron hole plasma Nature 4142869

[6] Kim K W, Pashkin A, Schäfer H, Beyer M, Porer M, Wolf T, Bernhard C, Demsar J, Huber R and Leitenstorfer A 2012 Ultrafast transient generation of spin density wave order in the normal state of $\mathrm{BaFe}_{2} \mathrm{As}_{2}$ driven by coherent lattice vibrations Nat. Mater. 11497501

[7] Brida D et al 2013 Ultrafast collinear scattering and carrier multiplication in graphene Nat. Commun. 41987

[8] Zewail A H 2000 Femtochemistry: atomic scale dynamics of the chemical bond J. Phys. Chem. A 104566094

[9] Sundström V 2008 Femtobiology Annu. Rev. Phys. Chem. 59 5377

[10] Keldysh L V 1964 Ionization in the field of a strong electromagnetic wave Sov. Phys. JETP 20130714

[11] Krausz F and Ivanov M 2009 Attosecond physics Rev. Mod. Phys. 81163234

[12] Backus S, Durfee C G III, Murnane M M and Kapteyn H C 1998 High power ultrafast lasers Rev. Sci. Instrum. 69 120723

[13] Bradler M, Baum P and Riedle E 2009 Femtosecond continuum generation in bulk laser host materials with sub $\mu \mathrm{J}$ pump pulses Appl. Phys. B 9756174

[14] Krauss G, Lohss S, Hanke T, Sell A, Eggert S, Huber R and Leitenstorfer A 2010 Synthesis of a single cycle of light with compact erbium doped fibre technology Nat. Photonics 4 336 
[15] Nisoli M, Silvestri S D and Svelto O 1996 Generation of high energy $10 \mathrm{fs}$ pulses by a new pulse compression technique Appl. Phys. Lett. 6827935

[16] Couairon A, Franco M, Mysyrowicz A, Biegert J and Keller U 2005 Pulse self compression to single cycle limit by filamentation in a gas with a pressure gradient Opt. Lett. 30 26579

[17] Cerullo G and De Silvestri S 2003 Ultrafast optical parametric amplifiers Rev. Sci. Instrum. 74118

[18] Riedle E, Beutter M, Lochbrunner S, Piel J, Schenkl S, Spörlein S and Zinth W 2000 Generation of 10 to $50 \mathrm{fs}$ pulses tunable through all of the visible and the NIR Appl. Phys. B 7145765

[19] Brida D, Manzoni C, Cirmi G, Marangoni M, Bonora S, Villoresi P, De Silvestri S and Cerullo G 2010 Few optical cycle pulses tunable from the visible to the mid infrared by optical parametric amplifiers J. Opt. 12013001

[20] Darginavičius J, Tamošauskas G, Piskarskas A, Valiulis G and Dubietis A 2012 Generation of tunable few optical cycle pulses by visible to infrared frequency conversion Appl. Phys. B 10817

[21] Brida D, Bonora S, Manzoni C, Marangoni M, Villoresi P, De Silvestri S and Cerullo G 2009 Generation of $8.5 \mathrm{fs}$ pulses at $1.3 \mu \mathrm{m}$ for ultrabroadband pump probe spectroscopy Opt. Express 17125105

[22] Ishii N et al 2005 Multimillijoule chirped parametric amplification of few cycle pulses Opt. Lett. 30 5679

[23] Vozzi C, Calegari F, Benedetti E, Gasilov S, Sansone G, Cerullo G, Nisoli M, De Silvestri S and Stagira S 2007 Millijoule level phase stabilized few optical cycle infrared parametric source Opt. Lett. 3229579

[24] Kraemer D, Cowan M L, Hua R, Franjic K and Miller R J D 2007 High power femtosecond infrared laser source based on noncollinear optical parametric chirped pulse amplification J. Opt. Soc. Am. B 248138

[25] Gu X et al 2009 Generation of carrier envelope phase stable 2 cycle $740 \mu \mathrm{J}$ pulses at $2.1 \mu \mathrm{m}$ carrier wavelength $O p t$. Express 17629

[26] Prandolini M J, Riedel R, Schulz M, Hage A, Hoeppner H and Tavella F 2014 Design considerations for a high power, ultrabroadband optical parametric chirped pulse amplifier Opt. Express 221594607

[27] Gale G M, Cavallari M, Driscoll T J and Hache F 1995 Sub $20 \mathrm{fs}$ tunable pulses in the visible from an $82 \mathrm{MHz}$ optical parametric oscillator Opt. Lett. 2015624

[28] Petrov V and Noack F 1996 Mid infrared femtosecond optical parametric amplification in potassium niobate Opt. Lett. 21 15768

[29] Nienhuys H K and Bakker H J 2008 Noncollinear optical parametric amplification in potassium titanyl phosphate pumped at $800 \mathrm{~nm} \mathrm{Appl.} \mathrm{Opt.} 4728703$

[30] Kraemer D, Hua R, Cowan M L, Franjic K and Miller R J D 2006 Ultrafast noncollinear optical parametric chirped pulse amplification in $\mathrm{KTiOAsO}_{4}$ Opt. Lett. 319813

[31] Rotermund F, Petrov V, Noack F, Pasiskevicius V, Hellström J and Laurell F 1999 Efficient femtosecond traveling wave optical parametric amplification in periodically poled $\mathrm{KTiOPO}_{4}$ Opt. Lett. 2418746
[32] Levenius M, Pasiskevicius V, Laurell F and Gallo K 2011 Ultra broadband optical parametric generation in periodically poled stoichiometric $\mathrm{LiTaO}_{3}$ Opt. Express 19 41218

[33] Liebel M, Schnedermann C and Kukura P 2014 Sub 10 fs pulses tunable from 480 to $980 \mathrm{~nm}$ from a NOPA pumped by an Yb:KGW source Opt. Lett. 3941125

[34] Limpert J, Aguergaray C, Montant S, Manek Hönninger I, Petit S, Descamps D, Cormier E and Salin F 2005 Ultra broad bandwidth parametric amplification at degeneracy Opt. Express 13738692

[35] Nikolov I, Gaydardzhiev A, Buchvarov I, Tzankov P, Noack F and Petrov V 2007 Ultrabroadband continuum amplification in the near infrared using $\mathrm{BiB}_{3} \mathrm{O}_{6}$ nonlinear crystals pumped at $800 \mathrm{~nm}$ Opt. Lett. 3233424

[36] Brida D, Marangoni M, Manzoni C, De Silvestri S and Cerullo G 2008 Two optical cycle pulses in the mid infrared from an optical parametric amplifier Opt. Lett. 3329013

[37] Brida D, Cirmi G, Manzoni C, Bonora S, Villoresi P, De Silvestri S and Cerullo G 2008 Sub two cycle light pulses at $1.6 \mu \mathrm{m}$ from an optical parametric amplifier $O p t$. Lett. 337413

[38] Wilhelm T, Piel J and Riedle E 1997 Sub 20 fs pulses tunable across the visible from a blue pumped single pass noncollinear parametric converter Opt. Lett. 2214946

[39] Cerullo G, Nisoli M and De Silvestri S 1997 Generation of $11 \mathrm{fs}$ pulses tunable across the visible by optical parametric amplification Appl. Phys. Lett. 7136168

[40] Baltuška A, Fuji T and Kobayashi T 2002 Visible pulse compression to $4 \mathrm{fs}$ by optical parametric amplification and programmable dispersion control Opt. Lett. 273068

[41] Huang S W, Moses J and Kärtner F 2012 Broadband noncollinear optical parametric amplification without angularly dispersed idler Opt. Lett. 3727968

[42] Nillon J, Crégut O, Bressler C and Haacke S 2014 MHz Tunable non collinear optical parametric amplifiers with pulse durations down to 6 fs Opt. Express 221496474

[43] Cirmi G, Brida D, Manzoni C, Marangoni M, De Silvestri S and Cerullo G 2007 Few optical cycle pulses in the near infrared from a noncollinear optical parametric amplifier Opt. Lett. 3223968

[44] Isaienko O and Borguet E 2013 Ultrabroadband few cycle infrared pulse generation from a noncollinear optical parametric amplifier based on bulk niobate crystals $J$. Opt. Soc. Am. B 30207580

[45] Isaienko O and Borguet E 2008 Generation of ultra broadband pulses in the near IR by non collinear optical parametric amplification in potassium titanyl phosphate Opt. Express 16 394954

[46] Cerullo G and Brida D 2013 Ultra broadband optical parametric amplifiers Ultrafast Nonlinear Optics (Berlin: Springer)

[47] Isaienko O and Borguet E 2009 Pulse front matching of ultrabroadband near infrared noncollinear optical parametric amplified pulses J. Opt. Soc. Am. B 2696572

[48] Manzoni C, Huang S W, Cirmi G, Farinello P, Moses J, Kärtner F X and Cerullo G 2012 Coherent synthesis of ultra broadband optical parametric amplifiers Opt. Lett. 37 18802 